\title{
NGC 7479 - the Rosetta stone of minor mergers?
}

\author{
S. Laine
}

\author{
University of Hertfordshire, United Kingdom
}

\section{Summary}

The overall morphology of the barred spiral galaxy NGC 7479 was modelled numerically in a simple minor merger scenario. I paid special attention to the morphology and features of the velocity field along the dominant spiral arm. The mass of the merging companion was $1 / 30$ of the total mass of the primary. The companion was started in a circular prograde orbit at six disk scale lengths from the centre of the primary. I followed the evolution of the merger until the companion had reached the nuclear region of the primary. A comparison between the modelled and observed morphologies of the stellar and the ionized and neutral gas distributions and velocity fields suggests that the transient look of NGC 7479 is a result of a minor merger. I also tested the effects of variations in several initial parameters of the merger. See Laine and Heller, 1999, for further details.

\section{References}

Laine, S. \& Heller, C. H., 1999, MNRAS, 308, 557 